\title{
Does Emotional Intelligence Fluctuate within Clinical Depressive Condition?
}

\author{
Yoon-Jung Kim, Eun-Hee Park ${ }^{\bowtie}$, Duk-in Jon, Myung Hun Jung, and Narei Hong \\ Department of Psychiatry, Hallym University Sacred Heart Hospital, Hallym University College of Medicine, Anyang, Republic of Korea
}

Objective Emotional intelligence (EI) is regarded an important factor related to psychiatric disorder. Most of previous studies of EI and depression only dealt with EI in present of depressive symptoms. Our study investigated EI both before and after remission of depression and to identify which component of $\mathrm{EI}$ is changeable by depression condition.

Methods EI was evaluated for 23 depression patients and 44 normal control participants. Adult Emotional Quotient Test (AEQT) was used as subjective EI measure and Emotional Literacy Test (ELT) was used as objective EI measure to both groups.

Results During the initial evaluation of the subjective EI, patient group reported significantly lower scores than control group on all AEQT sub-domains except 'empathy'. However, group difference on 'utilization' disappeared after the remission evaluation. There was no significant difference within the objective EI measure.

Conclusion According to the result of the study, EI could be sub-divided into trait-dependent EI and state-dependent EI. Further implication, strength and limitation were discussed.

Psychiatry Investig 2017;14(5):532-538

Key Words Emotional intelligence, Remission of depression.

\section{INTRODUCTION}

The emotional intelligence (EI) was first surfaced in the $1960{ }^{\prime}{ }^{1}$ and since has been a topic of vigorous research and Mayer et al. ${ }^{2}$ have become prominent researchers in this field. They suggest that EI is an ability to process the information about oneself and others' emotions and use the information in thought processing and behavior. ${ }^{3}$ They have also stated that EI consists of various aspects such as emotional perception/ identification, emotional facilitation of thought, emotional understanding, emotional management, and so on. ${ }^{4}$

EI not only serves an important role in health and adaptive lifestyle of ordinary people, ${ }^{5,6}$ but also is considered to be a crucial factor in explaining the cause of onset, characteristics of disorders, and outcomes in patients with psychiatric disorders. ${ }^{\text {? }}$ Especially, among the studies regarding depression and EI,

Received: September 7, 2016 Revised: November 30, 2016

Accepted: December 28, 2016 Available online: May 16, 2017

$\triangle$ Correspondence: Eun-Hee Park, MA

Department of Psychiatry, Hallym University Sacred Heart Hospital, Hallym University College of Medicine, 22 Gwanpyeong-ro 170beon-gil, Dongan-gu, Anyang 14068, Republic of Korea

Tel: +82-31-380-1854, Fax: +82-31-381-3753, E-mail: psypeh@hanmail.net

(a) This is an Open Access article distributed under the terms of the Creative Commons Attribution Non-Commercial License (http://creativecommons.org/licenses/bync/4.0) which permits unrestricted non-commercial use, distribution, and reproduction in any medium, provided the original work is properly cited. those studies regarding various sample populations such as adolescents, university students, and elderly consistently portray that those who have higher EI exhibit low levels of depression..$^{8-10}$

Previous depression and EI studies only deal with EI solely when the subjects are experiencing depression, which makes it hard to interpret the results as of whether the particular EI stems from a temporary state or from a vulnerable trait that the subjects were predisposed with.

Few studies looked into changes in EI depending on the severity of depression by comparing EI before and after the remission of depression. Nykliček et al. ${ }^{11}$ study sought into the relationship between EI and the symptoms depending on the process of treatment by observing patients diagnosed with mood and anxiety disorders. Their results showed that those patients who received cognitive behavioral treatment during hospitalization recovered their EI right after and 6 months after discharge from hospitalization. The recovery of EI was related to the alleviation of their symptoms of depression and anxiety. Hansenne et al. ${ }^{12}$ study also looked into change in EI in patients with depression resulting from remission of depression. It was confirmed that the patients with depression first showed significantly low scores in optimism/mood regulation and appraisal of emotion, while after the remission, the two 
sub-components showed recovery similar to that of the control group. This meant that at least certain sub-components within EI may be state dependent and that it may be necessary to target this area for depression treatment.

Nyklíček's and Hansenne's studies both have limitations in that they only used self-report questionnaire ${ }^{13,14}$ as means for measurement. Self-reported EI has a tendency to differ depending on the subject's sex ${ }^{15}$ or psychological health status. ${ }^{16}$ It is impossible to rule out participant's subjectivity. Subjectivity of the measurement always might be concern when utilizing self-reporting method. Patients with depression especially have a tendency to negatively distort recognition and interpretation of information, ${ }^{17}$ and it is difficult to ignore the possibility that they may make negatively biased reports about their own EI. Moreover, one study showed that self-reported based EI and objective evaluation based EI measurement differed substantially. ${ }^{18}$ Therefore, it is important to measure and review EI using multiple approaches by not only methods of self-report questionnaires, but also by methods that measure abilities using performance based evaluations. This study aims to observe the change in EI within pre- and postremission from depression using self-report and ability measurement methods. Hypotheses of the present study are following: 1) Compare to control group, depression patient would show lower EI. 2) After remission for depression, EI would be recovered which indicates its state-dependency.

\section{METHODS}

\section{Subjects}

Participants were men and women with ages ranging from 20 to 60 . At baseline, a total of 110 out and in patients diagnosed with depression visiting $\mathrm{H}$ Medical Center, located in Gyeonggi-do province, South Korea were recruited. All patients included in the study were diagnosed using the Korean Version of Mini International Neuropsychiatric Interview (KMINI) ${ }^{19}$ by clinical psychology trainees under the supervision of a clinical psychologist. The subjects with traumatic brain injury, bipolar disorders, psychotic disorders were excluded $(n=5)$. Five patients were excluded due to unreliable answers. Ninety patients (90\%) had major depressive disorder, 8 (8\%) were diagnosed with depressive disorder NOS and 2 (2\%) with dysthymic disorder. The comorbid axis I disorders other than depressive disorders were anxiety disorders $(n=29)$, alcohol related disorders $(n=2)$ and one anorexia nervosa. The recruitment period for the initial baseline assessment lasted from January 2011 to July 2013.

For a total 100 eligible candidates, the follow-up evaluation was performed after the five months from the initial evaluation which indicates average remission period from current pool.
Only 23 out of the 100 patients with depression participated in the second evaluation because the rest were either unavailable for the follow-up during treatment, refused to be engaged in the study, showed incomplete self-report questionnaires, or did not achieve a full remission from depression. Consequently, 23 cases that completed both the self-questionnaires and the objective tests of EI for both the baseline and the follow-up were included in the statistical analysis.

Psychological interventions (e.g., CBT) were not performed while participating in the study. All participants were in medication treatment only. The medications that participants were treated were following (the number of patients, doses): antidepressants: Escitalopram oxalate (2, 10-20 mg), Trazodone (11, 25-100 mg), Bupropion $\mathrm{HCl}(2,150-300 \mathrm{mg})$, anxiolytics: Buspirone $\mathrm{HCl}(12,5-20 \mathrm{mg})$, Alprazolam (9, 0.25-0.75 $\mathrm{mg}$ ), Lorazepam (5, 0.25-1 mg), antipsychotics: Quetiapine (3, 25-50 mg), Aripiprazole (3, 2-10 mg), hypnotics: Zolpi$\operatorname{dem}(5,10-12.5 \mathrm{mg})$.

The 45 normal participants with ages ranging from 20 to 60 years who did not have any psychiatric diagnosis by $\mathrm{K}_{-} \mathrm{MINI}^{19}$ and recorded less than 7 in K-HDRS were involved in this study as a control group. One normal participant who acquired more than 8 points of K-HDRS was excluded. The purpose of this study was well informed to all participants. The participants then agreed to take part in this research by voluntarily signing a written consent form, which was approved by the Institutional Review Board of H Medical Center.

\section{Measures}

\section{Mini International Neuropsychiatric Interview}

The Mini International Neuropsychiatric Interview (MINI) developed by Sheehan et al. ${ }^{20}$ is a structured clinical interview tool to evaluate Axis I psychiatric disorders based on DSM-IV \& ICD-10. The questions within this interview contain inclusion and exclusion criteria for psychiatric diagnoses. In the Korean validity study, 14 diagnoses, excluding 3 optional diagnoses out of the original total of 17 , had internal consistencies ranging from 0.42 to $0.91 .{ }^{19}$ Some of participants partially satisfied the full criteria of major depressive episode or dysthymia (e.g., duration of symptoms were classified into Depressive Disorder NOS).

\section{Korean Version of the Hamilton Depression Scale}

Hamilton $^{21}$ originally developed this scale to evaluate symptom severity and treatment outcome in depression. It contains 17 items measuring depressive symptoms over a past 7 days. The total possible scores of HDRS range from 0 to 52 . The Korean version of Hamilton Depression Rating Scale (KHDRS) was developed by Yi et al. ${ }^{22}$ with its reliability and va- 
lidity proven. Cronbach's alpha coefficient of the K-HDRS in the present study is 0.760 , which is an acceptable level.

\section{Adult Emotional Quotient Test}

Adult Emotional Quotient Test (AEQT) is a self-report questionnaire developed by $\mathrm{Moon}^{23}$ with 45 -items inventory categorized into the 5 factors based on the Salovey and Mayer ${ }^{4}$ model of EI. The 5 factors are 'perception', 'expression,' 'empathy', 'regulation', and 'utilization.' Perception is the ability to sensitively recognize and comprehend others' and one's own emotion. Expression is the ability for one to express one's own emotion precisely and distinguish emotions expressed by others. Empathy is ability to sense other peoples' emotions. Regulation is the ability to keep distance from others' emotion and show a reflective response. Utilization is the ability to understand subtle relations among emotions and interpret the meaning of the emotion. Each item is rated on a 1 to 5 response scale and the higher the score, the better the emotional intelligence. Cronbach's alpha coefficient of the AEQT in the present study is 0.855 which is fair enough.

\section{Emotional Literacy Test}

Emotional Literacy Test (ELT) is an objective test based on performance and was developed and validated by Ko and Kim. ${ }^{24}$ This test consists of two parts: emotion perception and emotion regulation. Each part is given the time limit of 10 minutes. Emotion perception comprises of 28 items measuring perception and identification of emotions from faces and three types of items were included: choosing an emotional word (i.g., anger, happy, sadness, etc.) matching with pictures of emotional facial expression in a multiple choice, choosing the most suitable facial expression for the given situation, and arranging facial expressions according to the degree of emotion they convey; from the strongest to the weakest. The emotion regulation is comprised of 12 items. Participants were asked to choose out of four responses one in given emotionevoked situation. The average total score of the emotional literacy test was 23, the mean score of the emotion perception was 17 , and the mean score of the emotion regulation was 6 .

\section{Procedure}

The patient group completed the baseline evaluation within two weeks after their first visit. Most of them were in the drugfree state. In the first evaluation, clinical psychology trainees conducted the structured clinical interview with K-MINI to diagnose clinical depression and K-HDRS ${ }^{22}$ to check their current emotional state. In incoming patient in second evaluation, we enrolled patients who had met following options: full remission reporting a K-HDRS score of 7 or less. The two types of EI measurement were administered to patients in two different time period (baseline, follow-up). Patients with depression received Emotional Literacy Test which was divided into two parts. Scores from each test were added for the total score. The Adult Emotional Quotient Test was also self-administered by patients. All evaluations for the control group were conducted only at baseline. The rest of procedures were the same with the patients group.

\section{Statistical analyses}

We conducted the independent $t$-test and $\chi^{2}$ test to identify the differences in the demographic characteristics between the patients and the control group. To see the differences in EI before and after the remission, we carried out a paired t-test. Independent t-test was conducted to compare the patients and the control group in their EI change represented by AEQT and ELT obtained before and after remission. All statistical analyses were conducted with SPSS 22.0 (IBM Corp., Armonk, NY, USA).

\section{RESULTS}

\section{Demographic characteristics}

Table 1 shows major demographic variables of the patient group and the normal control group. There were 25 males (36\%) and 43 females (64\%) participating in this study. The average year of age was 40.6 and the average year of education was 13.4. There were no significant demographical differences between two groups such as sex $\left[\chi^{2}(1, n=67)=0.893\right.$, ns), age [t $(64)=1.46, \mathrm{~ns}]$, and education $[\mathrm{t}(64)=-0.656$, ns]. Clinical characteristics of those patients with depression (4 inpatients, 24 outpatients) were of the following: average age for clinical onset was 39.8 years $(\mathrm{SD}=10.99)$, disease duration was an average

Table 1. Comparison between demographic variables of depression and control groups

\begin{tabular}{|c|c|c|c|c|c|c|}
\hline & Depression $(\mathrm{N}=23), \mathrm{M}(\mathrm{SD})$ & Control $(\mathrm{N}=44), \mathrm{M}(\mathrm{SD})$ & Total $(\mathrm{N}=67), \mathrm{M}(\mathrm{SD})$ & $\mathrm{t}$ & $x^{2}$ & $\mathrm{p}$ \\
\hline Gender* (\%) & & & & & 0.893 & 0.345 \\
\hline Male & $10(43)$ & $14(32)$ & $24(36)$ & & & \\
\hline Female & $13(57)$ & $30(68)$ & $43(64)$ & & & \\
\hline $\mathrm{Age}^{\dagger}$ & $43.48(10.84)$ & $39.16(12.69)$ & $40.64(12.18)$ & 1.46 & & 0.151 \\
\hline Years of education ${ }^{\dagger}$ & $13.09(2.27)$ & $13.47(1.20)$ & $13.34(2.08)$ & -0.656 & & 0.516 \\
\hline
\end{tabular}

*frequency (percent), ${ }^{\dagger}$ average (standard deviation) 
of 22 months ( $\mathrm{SD}=35)$, and $6(26 \%)$ out of a total of 23 patients had history of recurrent depressive episode.

\section{Comparison between before and after remission in the patient group}

Table 2 illustrates the change in severity of depression and EI. As for the change in severity of depressive symptoms from the baseline to the follow-up on K-HDRS, the K-HDRS score in second evaluation was significantly lower than that of the first evaluation, $\mathrm{t}(21)=-11.23, \mathrm{p}=0.000$. There were no significant differences in the total score and each subtest score of Emotional Literacy Test, t (21)=-0.76, ns; t (21)=-1.29, ns; $\mathrm{t}(21)=0.32$, ns. In AEQT, perception [ $\mathrm{t}(21)=3.10, \mathrm{p}=0.005$ ], expression $[\mathrm{t}(21)=3.77, \mathrm{p}=0.001]$, regulation $[\mathrm{t}(21)=3.91$, $\mathrm{p}=0.001]$, and utilization $[\mathrm{t}(21)=3.11, \mathrm{p}=0.005]$ as well as the total score, $t(21)=5.18, p=0.000$, showed significant improve- ment in the second evaluation compared to the first evaluation. Empathy was the only exception that showed no significant difference, $\mathrm{t}(21)=0.68$, ns. In other words, among the sub-factors of the self-report measure, emotion recognition, expression, regulation, and utilization improved with the remission from depression, and if this improvement would reach the levels of that of the normal group, it may implicate the state-dependent aspects of EI.

\section{Comparison of emotional intelligence between patients and normal control group}

Table 3 compares the scores taken at the baseline (T1) and at the endpoint (T2) in the severity of depression and EI of patients with depression to those of the control group. At T1, patients with depression showed significant difference in their severity of depression compared to that of the control

Table 2. Depression, anxiety, general intelligence, and emotional intelligence scores in the two study groups

\begin{tabular}{|c|c|c|c|c|c|}
\hline & $\begin{array}{c}\text { 1st evaluation } \\
(\mathrm{N}=23), \mathrm{M}(\mathrm{SD})\end{array}$ & $\begin{array}{l}\text { 2nd evaluation } \\
(\mathrm{N}=23), \mathrm{M}(\mathrm{SD})\end{array}$ & $\begin{array}{l}\text { Paired difference } \\
\text { (1st-2nd), M (SD) }\end{array}$ & $\mathrm{t}$ & $\mathrm{p}$ \\
\hline K-HDRS total & $18.52(5.90)$ & $4.04(2.31)$ & $-14.48(6.19)$ & -11.23 & $0.000^{* * *}$ \\
\hline ELT total & $22.87(4.88)$ & $22.39(5.26)$ & $-0.48(3.03)$ & -0.76 & 0.457 \\
\hline Perception & $17.74(3.95)$ & $17.13(4.15)$ & $-0.61(2.27)$ & -1.29 & 0.212 \\
\hline Regulation & $5.13(1.77)$ & $5.26(2.07)$ & $0.13(1.98)$ & 0.32 & 0.756 \\
\hline AEQT total & $124.70(10.53)$ & $136.26(12.73)$ & $11.57(10.72)$ & 5.18 & $0.000^{* * *}$ \\
\hline Recognition & $24.04(3.27)$ & $26.74(4.32)$ & $2.70(4.17)$ & 3.10 & $0.005^{* *}$ \\
\hline Expression & $20.22(3.33)$ & $22.65(2.77)$ & $2.44(3.10)$ & 3.77 & $0.001^{* * *}$ \\
\hline Empathy & $24.52(3.00)$ & $24.83(2.81)$ & $0.30(2.14)$ & 0.68 & 0.503 \\
\hline Regulation & $47.65(5.15)$ & $51.61(5.82)$ & $3.96(4.86)$ & 3.91 & $0.001^{* * *}$ \\
\hline Utilization & $8.26(3.83)$ & $10.43(3.07)$ & $2.17(3.35)$ & 3.11 & $0.005^{* *}$ \\
\hline
\end{tabular}

${ }^{* *} \mathrm{p}<0.01,{ }^{* * *} \mathrm{p}<0.001$. K-HDRS: Korean version of the Hamilton Depression Scale, ELT: Emotional Literacy Test, AEQT: Adult Emotional Quotient Test

Table 3. Difference between emotion intelligence and depression severity in the pre-remission/remission depression group and the control group

\begin{tabular}{lccccc}
\hline & 1st evaluation & 2nd evaluation & Control group & \multicolumn{2}{c}{$\mathrm{F}$} \\
\cline { 5 - 6 } & $(\mathrm{N}=23), \mathrm{M}(\mathrm{SD})$ & $(\mathrm{N}=23), \mathrm{M}(\mathrm{SD})$ & $(\mathrm{N}=44), \mathrm{M}(\mathrm{SD})$ & 1st-control & 2nd-control \\
\hline K-HDRS total & $18.52(5.90)$ & $4.04(2.31)$ & $0.80(1.49)$ & $37.537^{* * *}$ & $10.900^{* * *}$ \\
ELT total & $22.87(4.88)$ & $22.39(5.26)$ & $25.18(5.30)$ & $0.054^{*}$ & $0.543^{*}$ \\
$\quad$ Perception & $17.74(3.95)$ & $17.13(4.15)$ & $19.00(4.31)$ & 0.001 & 0.009 \\
Regulation & $5.13(1.77)$ & $5.26(2.07)$ & $6.18(2.12)$ & $3.145^{*}$ & 0.421 \\
AEQT total & $124.70(10.53)$ & $136.26(12.73)$ & $147.10(13.59)$ & $2.722^{* * *}$ & $0.147^{* *}$ \\
Recognition & $24.04(3.27)$ & $26.74(4.32)$ & $29.34(3.77)$ & $0.181^{* * *}$ & $0.872^{*}$ \\
Expression & $20.22(3.33)$ & $22.65(2.77)$ & $24.66(3.21)$ & $0.076^{* * *}$ & $0.788^{*}$ \\
Empathy & $24.52(3.00)$ & $24.83(2.81)$ & $25.18(3.36)$ & 0.095 & 0.324 \\
Regulation & $47.65(5.15)$ & $51.61(5.82)$ & $55.91(6.39)$ & $1.247^{* * *}$ & $0.080^{* *}$ \\
Utilization & $8.26(3.83)$ & $10.43(3.07)$ & $12.00(3.17)$ & & $1.403^{* * *}$ \\
\hline
\end{tabular}

${ }^{*} \mathrm{p}<0.05,{ }^{* *} \mathrm{p}<0.01,{ }^{* *} \mathrm{p}<0.001$. K-HDRS: Korean version of the Hamilton Depression Scale, ELT: Emotional Literacy Test, AEQT: Adult Emotional Quotient Test 
group. Although the severity of depression at T2 in patients with depression was significantly different from that of the control group, patients with depression were in full remission $(\mathrm{K}-\mathrm{HAMD}<7)$. Patients with depression did not show any significant differences in the total scores of ELT at T1 and T2 compared to that of the control group. Patients with depression did not show significant differences in the perception of ELT in T1 nor T2 compared to the control group. Albeit, at $\mathrm{T} 1$, patients with depression were significantly different from the control group regarding the regulation of ELT but not at T2; this may be interpreted as statistical chance finding. Patients with depression showed significant change in the total score and the sub-domain scores in AEQT, except for the empathy in AEQT. Patients with depression at T1 and T2 were not significantly different compared to the control group in scores for empathy in AEQT but were significantly different in scores regarding recognition, expression, and regulation in AEQT; this may implicate that these factors of EQ are traitdependent. Regarding the utilization score of AEQT, patients with depression were significantly different at $\mathrm{T} 1$ but reached the level of the control group at T2. This may implicate that this factor of EQ is state-dependent.

\section{DISCUSSION}

This study's objective was to investigate whether low EI in patients with depression is a stable and unchanging trait or is a changeable state depending on the clinical status of depression by examining the change in EI of patients before and after depression. According to the study's results, certain sub-components of EI in patients with depression can be within the trait dimension, while not all components are so, which implies the existence of a state-dependent factor, or emotion utilization.

In the objective EI measurement, emotion perception, there was no significant difference in group comparison and trial comparison in depression group. In the other hand subjective EI measurements showed difference at both group comparison and trial comparison in depression group. This difference in results could have explained by several interpretations. Firstly, depression patients are tend to have negatively biased self-perception which could be a reason that depression patients reported lower EI than normal group. Negatively biased self-schema in depression period could be recovered after the depression remission which made the difference of subjective EI between before and after remission for depression. In other words, depressive participants' actual EI ability could not be far behind that of the normal participants. Secondly, property of objective measurements materials could be the reason that we couldn't find any significant results in objective measurement. According to Liu's study, ${ }^{25}$ patients with depression are more sensitive to negative facial expressions than positive facial expressions. The emotions shown to be perceived in the objective ability measurement were mostly of negative contents such as anger, fear, and sadness. Since the task that was used in the present study composed of negative facial expression mostly, thus difference between depression and control group perhaps could be reduced.

Regarding analysis of objective ability measure on emotional regulation, because there was no significant difference within trials in depression group, difference between patient and normal control groups could be as result of statistical randomness. One the other hand, result of subjective measure on emotional regulation, there was significant difference within patients' EI. And in comparison between two groups, both trials of patient were significantly lower than normal group which implicates that subjective measure of emotional regulation is trait dependent sub-domain of EI.

Self-reported measurements of EI showed significant differences in the first and second evaluation regarding the other two sub-components of expression and utilization. While expression was not enhanced to the normal level, implying EI's trait dependent aspects, utilization was enhanced to the normal levels thus becoming the only component that hints EI's state dependent aspect. Hansenne et al. ${ }^{12}$ study showed utilization of emotions as an EI sub-domain that does not have significant differences before and after remission from depression thus conflicting with the results from our study. This conflict may stem from how the two studies measured different components of EI and differed in methodology. Schutte's scale ${ }^{26}$ used in Hansenne et al's study and AEQT used in our study both are developed based on Mayer and Salovey (1990)'s EI model. ${ }^{2}$ Although two scales share the same constructs about EI, they are different in their methods of measurement. Schutte's scale measures the degree to which people utilize their emotions in their lives with statements such as 'Emotions do not play a big part in how I deal with problems' and 'When my mood changes I see new possibilities.' Meanwhile, AEQT used in this study asks for the participants to choose one of the two solutions in a particular situation, for instance 'I lost a wallet my best friend gave me as a present. In this situation I [...]' and by doing so, makes the participants check a specific answer choice of how they utilize their emotions in a problem solving situation. AEQT used in the present study allows the participants to imagine more specific situations compared to the questionnaires for the utilization sub-domain of Schutte's scale, thus seems to be consisted of statements efficient to directly portray real life behaviors or tendencies.

This study's results show that 'empathy' did not show a significant difference for those experiencing depression compared to the healthy controls and it suggested that this EI 
sub-domain is not largely affected by depression. Similar to our study, some previous researches revealed that depression patients show no significant difference with healthy people in empathic abilities. ${ }^{27,28}$ This is contrary to some previous studies demonstrated that people with depression show lower empathic concern ${ }^{29}$ and limited perspective taking ability. ${ }^{30}$ However, this controversy could be results of differences in the definition of empathy and the methodologies that have used in EI measure. ${ }^{31}$ If we look into other studies that further subdivided EI in patients with depression, in cases where major depression and inability for emotion expression is accompanied, patients have a tendency to be unable to outwardly express their situation dependent empathetic decisions, ${ }^{32}$ and have difficulty in making the correct judgments on others' emotional state even though they have the ability to differentiate the emotions themselves. ${ }^{33}$ These types of previous studies may support the results from our study.

Those who experience psychiatric problems accompany various characteristics and degrees of functional deficits and hence require specialized clinical interventions. ${ }^{34}$ EI has shown its potential to be a prominent target for treatment which is one of the key factors to influence daily function and level of adaptation. ${ }^{35}$ For these reasons, the study that examines subdivided EI of depression patients may have an important significance in the clinical field. Our study found state dependent EI, utilization, recovered to the normal level and the sub-domain of EI seems to have the potential to be enhanced significantly with clinical intervention. If clinical intervention takes place targeting to treat the EI sub-domain that has a high possibility for improvement, we may expect a faster treatment effect, prevent relapse, and provide a psychological motivation for the treatment for the patients, which in turn will aid in continuing the psychological treatment.

This study has following strengths. First, this study implemented strict standards to diagnose depression using structured interviews and increased the diagnostic accuracy. The study also excluded various psychiatric diagnoses that included depressive symptoms and has mainly included those patients with major depressive disorder. Our research results regarding such comparatively homogeneous participants are thought to better represent EI characteristics of patients with major depression. Yet in a different aspect, it is still of question whether our study's result can be generalized to patients with other types of depression and requires further research.

This study conducted a longitudinal study by tracking the well retained patients with depression. This allowed for the changing patterns of EI before and after the patients' remission from depression. Since follow-up study was simultaneously made with a within subject design for the same patient group, effects of individual difference variables such as sex, age, and education levels were controlled to better identify the targets for this research.

As far as we understand, most studies observing the relationship between depression and EI have only included selfreport measurements. In our study we conducted objective measurements for EI that somewhat supplemented the limitation of subjective self-report bias.

It seems necessary for future studies to investigate EI that can be generally applied to diverse types of depression. Additionally, since this study only included two sub-categories of EI using objective ability measurements, researches need to include other sub-categories in the future. Finally, it is advisable to recruit diverse patients from various centers for a larger sample size that can allow for the results of study more plausible in becoming generalized.

We investigate the difference of emotional intelligence before and after the depression remission. The result from the present study support that EI could be divided into two domains: state dependent EI domain (e.g., utilization) and the trait dependent EI domains (e.g., recognition, expression, regulation). In other words, there are some changeable and stable EI subcomponents according to the depression severity. In the view of our results, changeable (state dependent) EI could be the first target of depression treatment.

\section{REFERENCES}

1. Leuner B. [Emotional intelligence and emancipation. A psychodynamic study on women]. Praxis der Kinderpsychologie und Kinderpsychiatrie 1966;15:196-203.

2. Mayer JD, DiPaolo M, Salovey P. Perceiving affective content in ambiguous visual stimuli: a component of emotional intelligence. J Pers Assess 1990;54:772-781.

3. Mayer JD, Salovey P, Caruso DR. Emotional intelligence: new ability or eclectic traits? Am Psychol 2008;63:503-517.

4. Salovey P, Mayer JD. What is Emotional Inteiiigence. Emotional Development and Emotional Intelligence: Educational Implications. New York: Basic Books; 1997.

5. Salovey P, Stroud LR, Woolery A, Epel ES. Perceived emotional intelligence, stress reactivity, and symptom reports: further explorations using the trait meta-mood scale. Psychol Health 2002;17:611-627.

6. Gohm CL, Corser GC, Dalsky DJ. Emotional intelligence under stress: useful, unnecessary, or irrelevant? Pers Individ Differ 2005;39:10171028.

7. Hertel J, Schütz A, Lammers CH. Emotional intelligence and mental disorder. J Clin Psychol 2009;65:942-954.

8. Lloyd SJ, Malek-Ahmadi M, Barclay K, Fernandez MR, Chartrand MS. Emotional intelligence (EI) as a predictor of depression status in older adults. Arch Gerontol Geriatr 2012;55:570-573.

9. Fernandez-Berrocal P, Alcaide R, Extremera N, Pizarro D. The role of emotional intelligence in anxiety and depression among adolescents. Individ Differ Res 2006;4:16-27.

10. Sulaiman SM. Emotional intelligence, depression and psychological adjustment among university students in the Sultanate of Oman. Int J Psychol Stud 2013;5:169-181.

11. Nyklíček I, Schalken P, Meertens S. The role of emotional intelligence in symptom reduction after psychotherapy in a heterogeneous psychiatric sample. Compr Psychiatry 2015;57:65-72. 
12. Hansenne M, Bianchi J. Emotional intelligence and personality in major depression: trait versus state effects. Psychiatry Res 2009;166:63-68.

13. Bar-On R. BarOn Emotional Quotient Inventory-Technical Manual. Toronto: Multi-Health System Inc; 1997.

14. Schutte NS, Malouff JM, Hall LE, Haggerty DJ, Cooper JT, Golden CJ, et al. Development and validation of a measure of emotional intelligence. Pers Individ Differ 1998;25:167-177.

15. Berrocal P, Extremera N. A review of trait meta-mood research. Adv Psychol Res 2008;55:17-45.

16. Martins A, Ramalho N, Morin E. A comprehensive meta-analysis of the relationship between emotional intelligence and health. Pers Individ Diff 2010;49:554-564.

17. Beck A. Conginitive Therapy and the Emotional Disorders New American Library. New York: A Meridian Book Psychology; 1976.

18. Goldenberg I, Matheson K, Mantler J. The assessment of emotional intelligence: a comparison of performance-based and self-report methodologies. J Pers Assess 2006;86:33-45.

19. Yoo S, Kim Y, Noh J, Oh K, Kim C, Namkoong K, et al. Validity of Korean version of the mini-international neuropsychiatric interview. Anxiety Mood 2006;2:50-55.

20. Sheehan DV, Lecrubier Y, Sheehan KH, Amorim P, Janavs J, Weiller E, et al. The Mini-International Neuropsychiatric Interview (MINI): the development and validation of a structured diagnostic psychiatric interview for DSM-IV and ICD-10. J Clin Psychiatry 1998:59(Suppl 20):22-33

21. Hamilton M. Development of a rating scale for primary depressive illness. Br J Soc Clin Psychol 1967;6:278-296.

22. Yi JS, Bae SO, Ahn YM, Park DB, Noh KS, Shin HK, et al. Validity and reliability of the Korean version of the Hamilton Depression Rating Scale (K-HDRS). J Korean Neuropsychiatr Assoc 2005;44:456-465.

23. Moon YL. Measuring Emotional Intelligence: A Case in Korea. Seoul: Samsung Life Social Mental Health Institute, Seoul National University Education Research Institute; 1996.

24. Ko Y, Kim J. The Norm and Interpretation Manual for Emotional Literacy Test. In press.
25. Liu WH, Huang J, Wang LZ, Gong QY, Chan RC. Facial perception bias in patients with major depression. Psychiatry Res 2012;197:217220.

26. Austin EJ, Saklofske DH, Huang SH, McKenney D. Measurement of trait emotional intelligence: testing and cross-validating a modified version of Schutte et al's (1998) measure. Pers Individ Dif 2004;36:555562.

27. Derntl B, Seidel EM, Schneider F, Habel U. How specific are emotional deficits? A comparison of empathic abilities in schizophrenia, bipolar and depressed patients. Schizophr Res 2012;142:58-64.

28. Thoma P, Zalewski I, von Reventlow HG, Norra C, Juckel G, Daum I. Cognitive and affective empathy in depression linked to executive control. Psychiatry Res 2011;189:373-378.

29. Cusi AM, MacQueen GM, Spreng RN, McKinnon MC. Altered empathic responding in major depressive disorder: relation to symptom severity, illness burden, and psychosocial outcome. Psychiatry Res 2011; 188:231-236.

30. Wilbertz G, Brakemeier EL, Zobel I, Härter M, Schramm E. Exploring preoperational features in chronic depression. J Affect Disord 2010;124: 262-269.

31. Schreiter S, Pijnenborg G, Aan Het Rot M. Empathy in adults with clinical or subclinical depressive symptoms. J Affect Disord 2013;150: $1-16$.

32. Hoffmann F, Banzhaf C, Kanske P, Gärtner M, Bermpohl F, Singer T. Empathy in depression: egocentric and altercentric biases and the role of alexithymia. J Affect Disord 2016;199:23-29.

33. Wolkenstein L, Schönenberg M, Schirm E, Hautzinger M. I can see what you feel, but I can't deal with it: impaired theory of mind in depression. J Affect Disord 2011;132:104-111.

34. Schütz A, Nizielski S. Emotional Intelligence as a Factor in Mental Health. Chemnitz (DE): Department of Psychology, Chemnitz University of Technology; 2012.

35. Campo M, Laborde S, Weckemann S, Colombus A. Emotional intelligence training: implications for performance and health. Adv Psychol Res 2015;101:75-92. 